\title{
I nuovi orizzonti dei metodi quantitativi
}

\section{Vittorio Capecchi}

Alma Mater Studiorum - Università di Bologna, Dipartimento di Scienze dell'Educazione doi: 10.7358/ecps-2014-009-cape_vittorio.capecchi@unibo.it

\section{NEW PERSPECTIVES IN QUANTITATIVE METHODS}

\section{Abstract}

In this essay, new perspectives in quantitative methods are analyzed by comparing linear causal models, used in the social research of Paul F. Lazarsfeld, and mathematical adaptive models with the models of Artificial Neural Networks (ANN) that have been promoted actually in Italy by Massimo Buscema and his Semeion Group of Rome. This analysis of quantitative methods is examined by considering three paradigms (Kuhn) of sociological research: (a) the paradigm of objectivity; (b) The paradigm of action-research; (c) The paradigm of feminist methodology. The essay presents a map of the relations between the three paradigms in terms of specificity/generality and a study on educational issue with the application of an ANN which enables a reflection on the relations between explanation and prediction. The difference between linear causal models and Artificial Neural Network are explained as: differences in linear/non-linear mathematical models, different possibilities of using variables at different levels of scale, different paradigms in social research, differences in the explanation-prediction relation, and as different use of time in the research process.

Keywords: Action-research, Artificial neural network, Feminist methodology, Linear causal models, Paradigm of objectivity.

Nel volume pubblicato per i quaranta anni della rivista che dirigo Quality and Quantity (dal titolo Applications of mathematics in models, Artificial Neural Networks and Arts) ho scritto un lungo saggio introduttivo ${ }^{1}$ in cui mi sono reso conto che la storia dei metodi quantitativi può essere capita solo se

\footnotetext{
1 Capecchi, 2010.
} 
viene interpretata insieme alle influenze che, nella ricerca sociologica, hanno avuto $\mathrm{i}$ "paradigmi» nel senso di Kuhn, cioè le "cornici» all'interno delle quali sono state collocare ricerche sociologiche considerate relativamente omogenee. Alla analisi dei cambiamenti avvenuti nei metodi quantitativi premetto perciò una descrizione dei tre paradigmi della ricerca sociologica individuati.

\section{I TRE PARADIGMI DELLA RICERCA SOCIOLOGICA}

Per definire questi paradigmi ${ }^{2}$ ho utilizzato le precisazioni date da Sandra Harding ${ }^{3}$ che definisce tre livelli all'interno di un "paradigma»: (a) Standpoint epistemology definita come «l'insieme delle relazioni che che collegano la conoscenza alla politica»; (b) Methodology, "una teoria o analisi di come la ricerca procede o dovrebbe procedere»; (c) Research methods, «tecniche (o modi di procedere) per accumulare prove [...]. In questo senso vi sono solo tre metodi di ricerca sociale: ascoltare o interrogare chi può dare informazioni; osservarne il comportamento; esaminare tracce storiche e documenti».

Sulla base di queste definizioni è possibile identificare nella storia della ricerca sociologica, almeno tre diversi "paradigmi»: il paradigma della oggettività, il paradigma della ricerca azione, il paradigma della Feminist methodology.

\subsection{Il paradigma dello oggettività}

Il paradigma della oggettività nei termini indicati da Sandra Harding può essere così formulato:

1. Standpoint epistemology: la ricerca sociologica ha come obiettivo quello di formulare teorie (spiegazioni) «scientifiche» analizzando in modo "oggettivo" atteggiamenti e/o comportamenti di persone.

2. Methodology: per raggiungere questo obiettivo occorre realizzare la massima distanza tra chi fa ricerca e i soggetti della ricerca in modo da avere risposte "oggettive» dai soggetti non influenzate dal ricercatore. I risultati della ricerca devono essere separati dalle azioni che potrebbero derivare dalle spiegazioni individuate dalla ricerca.

3. Research methods: i metodi di ricerca ammessi sono solo due: quelli in cui non c'è nessuna interazione del ricercatore con i soggetti della ricerca oppure quelli in cui l'interazione avviene attraverso una équipe di intervi-

2 Rinvio per una analisi più dettagliata a Capecchi, 2013.

3 Harding, 2004. 
statori professionisti preparati a svolgere «scientificamente» il loro lavoro garantendo l'oggettività dei risultati.

Come si può osservare rientrano in questo paradigma ricerche sociologiche molto diverse in quanto la «scientificità» di questo paradigma è concentrata solo in un punto: la relazione ricercatore/soggetti della ricerca. Tutte le ricerche basate su statistiche, tracce storiche, documenti ecc. rientrano in questo "paradigma» insieme alle ricerche che osservano i soggetti da una certa "distanza» oppure li intervistano attraverso le tecniche (ritenute scientifiche) della survey research. Questa distanza tra l'osservatore e i soggetti che vengono osservati viene osservata rigorosamente da ricercatori molto diversi come Lazarfeld e Goffman indipendentemente dalla loro minore o maggiore utilizzazione di metodi qualitativi o quantitativi.

Lazarsfeld (che indichiamo con la sigla PFL con cui era noto alla Columbia University), ad esempio, nella ricerca Die Arbeitslosen von Marienthal pubblicata nel 1932 insieme a Marie Jahoda e Hans Zeisel realizza una ricerca definita dai suoi autori una ricerca di immersione (sich einleben) e la scelta di non interagire con le persone di Marienthal per avere risultati più «oggettivi» fu, come scrisse PFL ${ }^{4}$, influenzata dalla lettura di Karl Marx. Per realizzare questo paradigma furono considerate sia caratteristiche individuali che di contesto e vennero effettuate analisi quantitative basate su statistiche ufficiali (statistiche della popolazione, reclami presentati alla Commissione industriale, risultati elettorali) e su altri tipi di dati (somme spese dalle persone nella cooperativa di consumo, numero di libri chiesti in prestito alla biblioteca comunale, numero abbonamenti alle riviste). Venne poi fatta una osservazione molto particolare: dietro l'angolo di una finestra con un orologio alla mano, si osservò a mezzogiorno il via vai delle donne e degli uomini sulla strada principale di Marienthal per quantificare, in una situazione di disoccupazione, la diversa lentezza degli uomini e delle donne che si avviavano a casa misurando il tempo delle soste di conversazione.

$\mathrm{Si}$ decise inoltre di osservare con metodologie qualitative il comportamento delle donne e degli uomini di Marienthal. Venne organizzato dalla équipe di ricerca un "Progetto vestiti» (il gruppo di ricerca distribuì 200 capi di vestiario alle famiglie più povere per il soccorso invernale del distretto e per giustificare questi regali chiese di rispondere ad alcune domande) e il «Progetto cure mediche» (il gruppo di ricerca accompagnò nelle loro visite una ostetrica ed una pediatra per dare, se necessario, gratuitamente i medicinali). Il gruppo di ricerca organizzò anche due corsi per le donne: un corso di disegno di modelli per riqualificare professionalmente le donne meno giovani e un corso di ginnastica per contattare le ragazze più giovani.

4 Lazarsfeld, 1986. 
Mi sono soffermato su questa ricerca per sottolineare che, all'interno del paradigma della "oggettività», la varietà metodologica può essere molto ampia e si passa da ricerche come quelle di PFL che utilizzano metodi prevalentemente quantitativi (ricerche che verranno ricordate nel paragrafo successivo) a ricerche come Asylums di Erving Goffman ${ }^{5}$ che aderisce al "paradigma della oggettività» presentandosi con il ruolo di istruttore di ginnastica per osservare chi vive nell'ospedale psichiatrico St. Elisabeth, non familiarizza con lo staffe trascorre la giornata riducendo al minimo le relazioni con i pazienti. Questo non gli impedisce di solidarizzare con loro e, come scrive nella introduzione, "Devo premettere che è probabile che il mio giudizio risenta del fatto di essere borghese: forse io ho sofferto per condizioni cui i pazienti di classe più povera sapevano far fronte con minor disagio [...] infine diversamente da quanto succede in alcuni pazienti, io arrivai in ospedale animato da ben scarso rispetto per la psichiatria in quanto scienza e per le altre entità ad essa collegate. [...] Se si vuole descrivere fedelmente la situazione del paziente non si può essere obiettivi» ${ }^{6}$.

All'interno di questo paradigma l'unico veto è che non si possono avere relazioni con i soggetti della ricerca per cui sono considerate «non scientifiche» ricerche come quelle basate sull'osservazione partecipante. Queste autolimitazioni non escludono però, all'interno di questo paradigma, la possibilità di realizzare ricerche politicamente impegnate.

\subsection{Il paradigma della ricerca azione}

Con il paradigma della ricerca azione chi fa ricerca si propone come obiettivo principale il cambiamento di atteggiamenti e comportamenti di un particolare aggregato di persone.

Il termine ricerca azione è stato utilizzato la prima volta da Kurt Lewin ${ }^{7}$ che così la definisce:

La ricerca azione [è] una ricerca che, confrontando le condizioni e gli effetti di varie forme di azione sociale e di ricerca, produce un'azione sociale. Una ricerca che produce solo libri non è sufficiente. Questo non significa in alcun modo che questo tipo di ricerca sia sotto tutti gli aspetti meno scientifica o «inferiore» a ciò che sarebbe richiesto da una scienza pura nell'area degli eventi sociali [...] [la ricerca azione si basa su] una spirale di passaggi ciascuno dei quali è formato da un processo ciclico di programmazione, azione e indagine su i risultati

Goffman, 1961.

Ivi, p. 26.

Lewin, 1946. 
dell'azione $[\ldots]$ la fase del programmare inizia in genere con qualche cosa di simile a un'idea generale. Per una ragione o l'altra si ritiene desiderabile raggiungere un dato obiettivo e non è del tutto chiaro circoscrivere esattamente questo obiettivo e definire i passi necessari per raggiungerlo. Il primo passo consiste nell'esaminare quest'idea con attenzione alla luce dei mezzi disponibili [...]. Il secondo passo consiste nell'eseguire le prime azioni della programmazione che è stata decisa [...]. Si passa poi a un processo ciclico di programmazione, esecuzione e conoscenza individuazione dei fatti necessari per valutare i risultati del secondo passo così da preparare le basi razionali per programmare il terzo passo o forse modificare tutto l'impianto della programmazione. ${ }^{8}$

La ricerca azione è quindi molto diversa dalle ricerche basate sul pradigma dell'oggettività ed è anche diversa dalle ricerche che utilizzano il metodo della osservazione partecipante. La "familiarità» e «l'intimità» tra chi fa ricerca e i soggetti della ricerca di cui parla William Foote Whyte avevano sempre come obiettivo la conoscenza ed anche Alfred Mc Clung Lee, che introduce il reader curato da Glenn Jacobs The partecipant observer ${ }^{9}$, scrive che:

L'osservazione partecipante è solo una via per superare le difficoltà di una più adeguata conoscenza sociale. Quello che accade quando si percorre quella via dipende dalla capacità e dalle interazioni dell'osservatore. Deve essere capace di vedere, ascoltare e mostrarsi sensibile alle interazioni di cui diventa una componente. ${ }^{10}$

L'obiettivo della ricerca azione non è invece solo quello della conoscenza ma è l'intreccio tra ricerca e azione che definisce questo tipo di paradigma.

Nelle ricerche basate sul paradigma della oggettività, e anche in quella basata sulla osservazione partecipante, si ha la sequenza: ipotesi $\rightarrow$ ricerca sul campo $\rightarrow$ analisi $\rightarrow$ conclusioni. Nella ricerca azione la sequenza è invece: Ricerca (ipotesi $\rightarrow$ ricerca sul campo $\rightarrow$ analisi) $\rightarrow$ Azione (azione $\rightarrow$ risultati $\rightarrow$ analisi) $\rightarrow$ Nuova ricerca (ipotesi $\rightarrow$ ricerca sul campo $\rightarrow$ analisi) $\rightarrow$ Nuova azione (azione $\rightarrow$ risultati $\rightarrow$ analisi).

$\mathrm{Si}$ prevede quindi un processo ciclico di ricerca $\rightarrow$ azione $\rightarrow$ nuova ricerca $\rightarrow$ nuova azione che si interrompe solo quando si sono raggiunti i cambiamenti desiderati dagli attori che hanno promosso la ricerca. Come ha precisato Kurt Lewin, "[la ricerca azione si compone] di una spirale di passaggi, ciascuno dei quali è formato da un processo ciclico di programmazione, azione e indagine sui risultati della azione»

Questo tipo di ricerca funziona solo se si realizza una collaborazione stretta tra i diversi tipi di attori (questa ricerca è stata anche definita co-ricerca)

8 Ivi, pp. 202, 205-206.

9 McClung Lee, 1970.

10 Ivi, p. 7. 
che sono coinvolti nel processo di ricerca e di azione. La ricerca azione nasce da un patto esplicito di collaborazione tra tre attori diversi: (a) chi coordina la ricerca e la équipe di ricerca; (b) altri attori pubblici o privati che sono coinvolti nel processo di cambiamento desiderato; (c) i soggetti della ricerca. Ed è questa collaborazione tra tre tipi di attori, che si propongono un cambiamento di atteggiamenti e comportamenti di persone o di una particolare situazione, che permette di: (i) raggiungere le conoscenze necessarie per formulare nuove teorie; (ii) individuare le azioni necessarie per il cambiamento.

Il muoversi al di fuori del paradigma della oggettività non significa che in questo tipo di ricerca sia rifiutato ogni tipo di controllo scientifico delle tappe della ricerca. La scientificità viene cercata attraverso una riflessione continua sulle diverse tappe della ricerca e su i comportamenti e atteggiamenti di tutti gli attori (compreso chi coordina la ricerca). È quindi una ricerca autoriflessiva (self-reflective) e Kurt Lewin, che analizza come esempio una ricerca azione di più attori che si proponevano di ridurre gli atteggiamenti razzisti nello stato del Connecticut, così descrive il procedere degli osservatori incaricati ogni giorno di trascrivere ciò che avviene nelle tappe della ricerca in modo da essere consapevoli, in modo il più completo possibile, di ciò che sta accadendo e permettere una valutazione presa collettivamente:

Il metodo di registrare gli eventi essenziali di chi partecipa al workshop comportò una sessione di valutazione alla fine di ogni giorno. Gli osservatori che avevano partecipato alle sessioni dei diversi sottogruppi, utilizzando un registratore, discussero sul tipo di leadership che avevano osservato, sul progresso o sulla mancanza di progresso nello sviluppo del gruppo che da un conglomerato di singoli individui attivi arriva ad essere un «noi» integrato e così via. ${ }^{11}$

All'interno di questa sequenza di ricerca-azione i metodi di ricerca possono essere qualitativi o quantitativi ma, a differenza di chi aderisce al paradigma della oggettività, non ci sono preclusioni nei confronti di nessun metodo di ricerca.

Il paradigma della ricerca azione può essere definito da questi punti:

1. Standpoint epistemology: la ricerca ha come obiettivo il cambiamento di atteggiamenti e comportamenti di persone oppure di una particolare situazione.

2. Methodology: per raggiungere questo obiettivo la ricerca prevede al suo interno le azioni necessarie per questo cambiamento e queste azioni sono decise in base alle analisi derivate dalla attività di ricerca. È una sequenza di ricerche-azioni basate sulla collaborazione tra chi fa ricerca, gli attori coinvolti, i soggetti della ricerca. La garanzia di scientificità è legata alla

11 Lewin, 1946, p. 210. 
capacità della ricerca di essere autoriflessiva con la presenza di osservatorì che svolgono un lavoro di osservazione-trascrizione permettendo agli attori la valutazione di ciò che sta accadendo.

3. Research methods: sono ammessi tutti i metodi di ricerca, sia quantitativi che qualitativi, purché all'interno della metodologia sopra indicata.

\subsection{Il paradigma della "Feminist methodology"}

Il paradigma della Feminist methodology (lascio il nome in inglese perché questa metodologia si è sviluppata soprattutto in area anglosassone) definisce uno stretto legame tra chi opera all'interno di questo paradigma e le azioni e teorie del movimento femminista ed è questo legame che rende distante chi fa questo tipo di ricerca non solo da chi opera all'interno del paradigma della oggettività ma anche da chi opera all'interno del più ampio paradigma della ricerca azione ${ }^{12}$.

Il movimento femminista è un soggetto politico collettivo eterogeneo che, in luoghi e tempi diversi, ha realizzato azioni diverse e proposto teorie femministe diverse. Nonostante queste diversità Caroline Ramazanoglu e Janet Holland ${ }^{13}$ scrivono che quando una ricercatrice utilizza una metodologia femminista per realizzare le sue ricerche a favore di altre donne fa riferimento a una "comunità femminista epistemica»:

Una comunità epistemica è un termine che identifica una collettività costruita socialmente con delle regole condivise che autorizza a parlare come un particolare tipo di soggetto esperto [...]. Il femminismo esiste come comunità epistemica immaginata nel senso che non è necessario incontrarsi insieme per esistere come collettività e non si tratta semplicemente di un'aggregazione di donne; ciò che le donne o le esperte in femminismo hanno oggi in comune è comunque di essere aperte all'indagine. ${ }^{14}$

Per capire le direzioni della metodologia femminista si può partire dalla relazione tra oggettività/soggettività. Questa relazione, come scrivono Caroline Ramazanoglu e Janet Holland ${ }^{15}$ va vista all'interno della relazione conoscen$\mathrm{za} / \mathrm{verità} \mathrm{che} \mathrm{permette} \mathrm{di} \mathrm{individuare} \mathrm{un} \mathrm{continuum} \mathrm{tra} \mathrm{due} \mathrm{posizioni} \mathrm{estreme}$ che possono essere prese da chi fa ricerca: (a) Verità assoluta (questa posizione

12 Per una collocazione della Feminist methodology all'interno degli altri metodi rinvio a Capecchi, 1996.

13 Ramazanoglu \& Holland, 2002, pp. 138-139.

14 Ibidem.

15 Ivi, p. 62. 
è presa da chi ritiene di essere «un osservatore che sa tutto e osserva dall'esterno ed è indipendente da ciò che deve essere osservato»); (b) Relativismo assoluto («è la posizione di chi non individua nessun modo per scegliere tra diverse versioni della verità»).

Tra queste due posizioni estreme la metodologia femminista si colloca in una posizione intermedia perché ammette che è sempre possibile considerare alcune interpretazioni migliori di altre e d'altra parte non è possibile arrivare a una «verità assoluta» che sia al di fuori delle relazioni che si stabiliscono tra le donne che fanno ricerca e le donne soggetti della ricerca. Quali sono infatti i rischi di chi non sceglie la posizione intermedia? Il problema è posto con chiarezza da Sandra Harding ${ }^{16}$ :

Se la comunità di ricercatori e critici «qualificati» esclude sistematicamente, ad esempio, le afroamericane e le donne di tutte le razze e se la cultura più ampia è stratificata per razza e genere e perde la capacità critica di questa stratificazione, non è plausibile immaginare che i valori e gli interessi razzisti e sessisti dovrebbero essere identificati all'interno di una comunità di scienziati composta interamente da persone che - intenzionalmete o meno - beneficiano di questo razzismo e sessimo.

Di fronte a questa situazione i seguaci dell'oggettivismo ipotizzano una «neutralità rispetto ai valori» che può avere effetti ancora più negativi:

L'oggettivismo concettualizza fin troppo ampiamente la desiderata neutralità rispetto ai valori dell'oggettività. Chi segue l'oggettivismo chiede l'eliminazione del processo di ricerca e dai risultati della ricerca di tutti i valori e interessi presenti nella società. È chiaro, tuttavia, che non tutti i valori e interessi sociali possono avere gli stessi effetti negativi sui risultati della ricerca. Ad esempio i valori di una democrazia più avanzata hanno prodotto sistematicamente credenze meno parziali e distorte rispetto ad altri possibili valori. ${ }^{17}$

L'aderire a valori distorti non è certamente la posizione di Goffman o di Lazarsfeld in ricerche come Die Arbeitlosen von Marienthal oppure The academic mind né è quella di molte ricerche fatte con il paradigma della ricerca azione. Ciò che emerge dalle precisazioni di Caroline Ramazanoglu e Janet Holland oppure di Sandra Harding è che i tre paradigmi analizzati (quello della oggettività, della ricerca azione oppure della ricerca azione) sono tutti e tre delle posizioni intermedie che differiscono tra di loro per le diverse modalità con cui viene ricercata una "maggiore oggettività».

16 Harding, 2004, p. 55.

17 Ivi, p. 56. 
La maggiore oggettività della metodologia femminista è definita da Sandra Harding ${ }^{18}$ "oggettività forte»:

L'oggettività forte dovrebbe precisare la strategia per definire le assunzioni sociali che a) influenzano la ricerca nelle fasi di identificazione e concettualizzazione dei problemi scientifici e formazione delle ipotesi (il "contesto della ricerca»); b) tendono ad essere ripartite tra gli osservatori legittimati e scelti per la ricerca e si tratta di valori e interessi «collettivi» e non individuali; c) tendono a strutturare le istituzioni e gli schemi concettuali delle discipline. Queste procedure sono anche capaci di d) distinguere tra quei valori e interessi che portano alla produzione di proposizioni sulla natura e relazioni sociali che sono meno parziali e distorte («meno falsi») da valori come correttezza, onestà, libertà e potremmo aggiungere quelli della democrazia più avanzata che li alimenta. ${ }^{19}$

\section{Questa oggettività forte richiede una forte riflessività (strong reflexivity):}

Una oggettività forte richiede che i soggetti della conoscenza siano posti sullo stesso piano critico e causale degli oggetti della conoscenza. La forte oggettività richiede ciò che noi definiamo come una "forte riflessività». Questa è necessaria perché è evidente che ciò in cui si crede in base a una data cultura incide in ogni fase dell'inchiesta scientifica: nella selezione dei problemi, formazione di ipotesi, disegno della ricerca (inclusa l'organizzazione delle comunità di ricerca), raccolta dei dati, decisione di quando interrompere la ricerca, modalità cui cui sono diffusi i risultati della ricerca. ${ }^{20}$

La riflessività forte di cui parla Sandra Harding è realizzata da «osservatori scelti come legittimi» e questi osservatori non si occupano solo di metodi ma di valori politici che devono essere rispettati durante tutte le fasi della ricerca.

Le ricerche che seguono la metodologia femminista possono utilizzare o non utilizzare ricerche basate sulla ricerca azione. In un manuale scritto da Shulamit Reinharz ${ }^{21}$ dal titolo Feminist methods in social research sono indicati questi metodi:

Ricerca femminista basata su interviste, etnografia femminista, ricerca femminista basata sulla survey e altri tipi di ricerca utilizzanti metodi quantitativi, ricerca sperimentale femminista, ricerca femminista interculturale, ricerca femminista di storia orale, analisi femminista di contenuti, studi di caso femministi, ricerca azione femminista, ricerca femminista utilizzante più metodi.

\footnotetext{
8 Harding, 1992.

Ivi, p. 580 .

Harding, 2004, p. 55.

Reinharz, 1992.
} 
La ricerca azione femminista è quindi solo uno dei metodi della metodologia femminista. Il paradigma della metodologia femminista può essere perciò definito in questi punti:

1. Standpoint epistemology: la ricerca ha come punto di riferimento le teorie e le lotte espresse dai movimenti femministi. Chi fa ricerca si confronta con una "comunità femminista epistemica».

2. Methodology: per raggiungere questo obiettivo la ricerca parte da una relazione privilegiata tra le donne che fanno ricerca e le donne che sono soggetti della ricerca e le spiegazioni individuate sono ritenute valide se tengono conto dei valori espressi dalla comunità femminista epistemica che considera importante valorizzare e incrementare il potere di tutte le donne. Una maggiore oggettività è ottenibile se la ricerca prevede al suo interno "osservatori legittimati» che devono tener conto non solo della correttezza dei metodi ma anche della correttezza con cui i valori femministi sono stati seguiti in tutte le fasi della ricerca. È una ricerca autoriflessiva.

3. Research methods: sono ammessi tutti i metodi, sia quantitativi che qualitativi, purché all'interno della metodologia sopra indicata.

\subsection{La mappa dei tre paradigmi e i metodi quantitativi}

Quali sono le relazioni tra i tre paradigmi in termini di generalità/specificità? Immaginiamo di disegnare una mappa dei tre paradigmi utilizzando i diagrammi di Venn dell'insiemistica. 11 paradigma della oggettività nasce come contrapposizione negli anni trenta tra due scuole sociologiche statunitensi: quella di PFL a New York e quella di ecologia sociale urbana di Chicago. Il paradigma dell'oggettività viene codificato da PFL che identifica la scientificità nella massima distanza tra chi fa ricerca e i soggetti che diventano "oggetto" di questa analisi. Se si vuole disegnare una mappa si può iniziare con il disegnare due diagrammi di Venn che si intersecano di cui uno è rappresentato da (A) Ricerche realizzate con il paradigma dell'oggettività e l'altro da Ricerche realizzate con i metodi qualitativi della scuola di Chicago con una zona di intersecazione perché anche nella scuola di Chicago si producevano ricerche che soddisfacevano i requisiti della oggettività di PFL (ad esempio le ricerche basate sull'analisi di diari, materiali di tipo storico ecc.).

Se si passa a (B) Ricerche realizzate con il paradigma della ricerca azione la distanza tra chi fa ricerca e i soggetti della ricerca non è più considerato un criterio discriminante e quindi il diagramma di Venn che considera questo secondo paradigma ingloba i due precedenti inserendovi le caratteristiche, pri- 
ma ricordate, della ricerca azione. Se poi si passa a (C) Ricerche realizzate con la Feminist methodology è del tutto evidente che vengono introdotti vincoli aggiuntivi rispetto a quelli più generali della ricerca azione per cui il diagramma di Venn di questo terzo paradigma ingloba tutti i diagrammi precedenti.

La mappa complessiva dei tre paradigmi è rappresentabile in una figura in cui c'è un cerchio più ampio (il paradigma $\mathrm{C}$ ) che ingloba un cerchio minore (il paradigma B) al cui interno si possono inserire due cerchi più piccoli che si intersecano di cui uno è il paradigma $A$.

La relazione tra questi tre paradigmi e i due tipi di metodi quantitativi che saranno presentati nel paragrafo successivo (i modelli causali lineari e i modelli matematici adattivi) sembrerebbe a prima vista impropria perché nei tre paradigmi delle ricerca considerati non viene mai presa in esame la differenza tra metodi quantitativi e metodi qualitativi.

In realtà le connessioni tra i tre paradigmi e i due tipi di metodi quantitativi considerati sono elevate perché c'è una filosofia nei due metodi matematici che verranno esposti che li avvicina diversamente ai tre paradigmi. In sintesi si può infatti affermare che i modelli causali lineari sono più vicini al paradigma della oggettività mentre i modelli matematici adattivi sono più vicini al paradigma della ricerca azione e a quello della Feminist methodology.

La vicinanza dei modelli causali lineari al paradigma della oggettività non dovrebbe sorprendere perché sono stati presentati nella loro coerenza interna dallo stesso autore PFL. I modelli causali lineari (basati su una variabile dipendente che viene spiegata (causalmente) da variabili intervenienti e indipendenti sono la trasposizione matematica dell'idea del paradigma della oggettività che prende le distanze dal modo di sentire più intenso e profondo dei soggetti (modo di sentire che solo interviste in profondità potevano far emergere) per "oggettivare» e spiegare i loro comportamenti e atteggiamenti attraverso le proposte dei modelli causali lineari.

Con il secondo e terzo paradigma si esce dalla finalità complessiva di «spiegare» un comportamento o un atteggiamento per dare importanza a una relazione ricerca/azione in cui la finalità complessiva è raggiungere un "obiettivo": come cambiare una data situazione (e non, solo, come spiegarla). I modelli matematici adattivi come le ANN corrispondono maggiormente a questi due diversi paradigmi perché, nella loro struttura matematica si propongono più di "prevedere» che di «spiegare» e la previsione di raggiungere un dato obiettivo è strettamente inseribile in un tipo di ricerca che si proponga di seguire il secondo o il terzo paradigma. È del tutto evidente che le ricerche che seguono il secondo e terzo paradigma non è assolutamente detto che seguano i modelli matematici delle ANN; è però vero che se si è di fronte ad una applicazione delle ANN si è quasi sicuramente all'interno del secondo o del terzo paradigma. 


\section{I CAMBIAMENTI NEI METODI QUANTITATIVI}

Per capire la storia dei cambiamenti nei metodi quantitativi dai modelli causali lineari proposti da PFL ai modelli non lineari basari sulle reti neurali artificiali occorre tener conto di almeno tre spostamenti: (a) un cambiamento tecnico basato sulle nuove conquiste dei modelli matematici e dei software relativi; (b) un cambiamento nei paradigmi, cioè nella possibilità di collocarsi consapevolmente all'interno di paradigmi della ricerca sociologica diversi; (c) un cambiamento culturale avvenuto nella filosofia sottostante I modelli matematici.

\subsection{I modelli causali lineari di P.F. Lazarsfeld}

Lo scenario dei metodi quantitativi presentato da $\mathrm{PFL}^{22}$ è uno scenario che si muove all'interno di tre limiti: (a) sul piano tecnico i modelli matematici proposti da PFL non riescono a mettere insieme variabili al livello nominale, insieme a variabili al livello della scala ordinale o di rapporti e nel complesso non si esce dalle relazioni lineari tra variabili indipendenti, intervenienti e dipendenti; (b) sul piano dei paradigmi non si esce dal paradigma della oggettività prima descritto; (c) sul piano della filosofia sottostante si aderisce all'individualismoi metodologico e al positivismo (l'accenno, prima ricordato, di PFL a Marx è, sotto questo aspetto, significativo).

Ho intitolato questo paragrafo «i modelli causali lineari», anche se PFL ha proposto modelli più complessi come l'analisi della struttura latente ${ }^{23}$, perché la filosofia complessiva di PFL non esce da questi modelli lineari e vede i comportamenti e gli atteggiamenti delle persone come un insieme di variabili tra le quali selezionare la variabile dipendente (quel dato tipo di consumo o di comportamento elettorale) spiegata causalmente da un insieme di variabili intervenienti (atteggiamenti, comportamenti ecc.) e con, alla periferia del modello, delle variabili indipendenti (distinzione uomo/donna, età, classi sociali, caratteristiche del contesto in cui le persone si muovono ecc.). Questo modo di procedere sarà sviluppato da Hubert Blalock ${ }^{24}$ ma nelle sue linee generali cì̀ già tutto negli negli scritti di Lazarsfeld.

All'interno di questo tipo di analisi PFL si è mosso molto bene proponendo due strategie: (a) quella di considera $\mathrm{i}$ «casi devianti» rispetto alle tendenze principali (ad esempio, nelle sue analisi del comportamento elettorale

22 Rinvio a tre miei saggi su Lazarsfeld: Capecchi, 1967, 1985, 1999.

23 Sulla analisi delle struttura latente rinvio a Lazarsfeld \& Neal, 1968 e Neil, 1999.

24 Blalock, 1985. 
data la tendenza a votare democratico da parte delle persone più povere, nere, cattoliche ecc.) PFL si interroga su che cosa accade quando una persona è sia nero che ricco e così via dimostrando che quando vi sono sulle stesse persone delle cross pressures il risultato è un rallentamento nel prendere decisioni; (b) l'altro tipo di proposta di PFL è stata quella di considerati i diversi cerchi di influenza rispetto alla decisione. C'è il nucleo familiare ma ci sono anche gli opinion leader e così via e il processo per prendere decisioni non è spiegato da variabili intervenienti e indipendenti rilevate da una ricerca basata su interviste (la ricerca analizzata con metodi quantitativi si limita a verificare $\mathrm{i}$ due o più stadi della comunicazione che sono stati ipotizzati dal ricercatore $\mathrm{e}$ che sono basati sulla sua capacità di osservare e proporre spiegazioni).

Il risultato è che ancora oggi i libri di PFL sono alla base di analisi del comportamento elettorale in saggi di interessante valore metodologico come quello di Cristiano Vezzoni e Moreno Mancosu ${ }^{25}$ che, partendo da due ricerche del comportamento elettorale di PFL con l'aggiunta dei contributi di Granovetter sulla "forza dei legami deboli» ${ }^{26}$, interpretano con metodi quantitativi il successo del Movimento cinque stelle nelle elezioni del 2013.

Ancora due riflessioni: una su i limiti matematici e concettuali dei modelli causali lineari e una sulla relazione tra ricerca e valutazione politica indipendentemente dai metodi utilizzati e dalla validità delle interpretazioni teoriche.

Su i limiti dei modelli causali posso riferirmi ad una esperienza personale. Ho applicato i modelli causali lineari molti anni fa a due ricerche promosse dall'Istituto Cattaneo di Bologna: Il comportamento elettorale italiano e L'attivista di partito ${ }^{27}$ e sono modelli che funzionano anche con dati molto diversi: ne Lattivista di partito si trattava di dati relativi a un piccolo campione di attivisti comunisti e democristiani di cui erano disponibili lunghe interviste qualitative che avevo interpretato con variabili quantitative; ne Il comportamento elettorale in Italia si trattava invece di dati quantitativi relativi ai voti espressi nei comuni italiani. In entrambi i casi i modelli causali lineari «funzionarono bene» sia nell' individuare una tipologia di attivisti non rilevabile dalle interviste qualitative sia nel permettere confronti tra le diverse variabili che compongono le tipologie.

Restano comunque limiti molto consistenti: (a) non sempre è ragionevole proporre delle relazioni causa effetto tra le variabili che caratterizzano diversi soggetti (ad esempio con questi modelli si assume che sono gli atteg-

25 Vezzoni \& Mancosu, 2013. Il testo è disponibile in Rete.

26 Lazarsfeld, Berelson, \& Gaudet, 1944; Berelson, Lazarsfeld, \& Mc Phee, 1954; Granovetter, 1973.

27 Alberoni, 1967; Galli, 1968. 
giamenti a "causare» un dato comportamento ma vi sono studi accreditati di psicologia sociale che mostrano come la relazione atteggiamenti/comportamento sia più complessa e vi possano essere consistenti feedback); (b) vi possono essere relazioni tra variabili non lineari (ad esempio in corrispondenza a diverse fasce di età vi possono essere atteggiamenti e comportamenti che non seguono logiche lineari); (c) questo tipo di modellistica non permette di analizzare flussi di influenza e formazione di nuovi oggetti collettivi essendo per questo tipo di situazioni necessario l'utilizzo di modelli di simulazione ${ }^{28}$.

Sul secondo punto mi limito a considerare due ricerche di PFL che ebbero valutazioni (politiche) molto diverse.

La ricerca Personal influence, pubblicata nel 1955 da PFL insieme a Elihu Katz ${ }^{29}$, fu finanziata dall'Ufficio Ricerche della Macfadden Publications per capire come doveva organizzare le sue campagne pubblicitarie. Il metodo utilizzato fu quello della survey research e con questa ricerca Lazarsfeld verificò la teoria dei two step flow of communication per cui la maggioranza delle donne, nelle loro decisioni di consumo, non è direttamente influenzata dai mass media ma da una rete di leaders di opinione che fanno parte della loro rete di relazione. La ricerca fu però valutata negativamente perché il 1955 è noto, negli Stati Uniti come l'anno di Martin Luther King ${ }^{30}$ alle cui iniziative aderirono docenti e studenti della Columbia University. Non sorpresero quindi le valutazioni molto critiche che fecero a Lazarsfeld sia i due Lynd che giovani studenti radicali come Seymour Martin Lipset. La ricerca Personal influence segnalò così due problemi: (a) la scelta di fare una ricerca su alcune tematiche non è mai, in un dato contesto storico, una scelta neutrale (indipendentemente dal paradigma utilizzato); (b) se si sceglie come tema quello della influenza dei mass media sulle persone è diverso studiare questi effetti per agevolare le campagne dei pubblicitari oppure capire i meccanismi di potere presenti nelle emissioni televisive che in quel periodo diffondevano contenuti razzisti, militaristi e sessisti.

28 Su questo tipo di modellistica rinvio a Capecchi, 2010. Sui modelli di simulazione rinvio in particolare all'opera di Conte, Hagelmann, \& Terna, 1997.

29 La ricerca è disponibile in lingua italiana con il titolo L'influenza personale in comunicazione (2012).

30 In quell'anno infatti a Montgomery, in Alabama, Rosa Parks, una signora di mezza età, decise di ribellarsi al segregazionismo nei confronti dei neri che li obbligava a sedersi negli autobus negli ultimi posti anche se i primi posti erano liberi. Rosa Parks si sedette nei primi posti vuoti di un autobus di Montgomery e per questo gesto fu riconosciuta colpevole e condannata a pagare una multa di dieci dollari. Questo gesto spinse Martin Luther King (che nel 1955 aveva ricevuto il Ph.D. in Teologia all'Università di Boston) a presentarsi come leader del movimento pacifico per i diritti dei neri negli Stati Uniti iniziando il boicottaggio degli autobus che portò nel 1956 a una sentenza della U.S. Supreme Court che definiva incostituzionale la segregazione dei neri negli autobus. 
Le valutazioni contro Lazarsfeld cambiarono quando uscì la ricerca The academc mind (1958) scritta con Wagner Thielens che analizzava il maccartismo nelle università americane. La ricerca fu finanziata da The Found for the Republic, una emanazione della Ford Foundation controllata, all'epoca, da intellettuali progressisti legati a John Kennedy e poté utilizzare un finanziamento molto elevato: un quarto di milione di dollari con la possibilità di realizzare un campione stratificato consistente (2451 docenti intervistati, di cui l'11\% donne, in 165 Colleges universitari), utilizzando due società di intervistatori/intervistatrici ed una équipe, coordinata da David Riesman, per controllarle e riflettere su questa esperienza.

In questa ricerca è molto interessante l'uso della matematica e delle analisi quantitative come "difesa» nei confronti degli attacchi che la potente destra americana avrebbe potuto fare ai risultati della ricerca. Venne infatti scritto nel libro un capitolo secondo che utilizzava solo risposte qualitative che permisero, e permettono ancora, di documentare con grande dettaglio le violenze del maccartismo subite dai docenti delle università americane e la condanna di queste violenze è fatta dai due autori senza mezzi termini. Negli altri capitoli però c'è un grande sfoggio di modelli matematici come l'analisi della struttura latente e venne utilizzata una terminologia neutrale (ad esempio si utilizzò il termine permissive invece che liberal or progressive contrapposto al termine conservative) per cui la ricerca di "oggettività» non fu solo cercata nella "neutralità» della équipe di intervistatori e intervistatrici ma anche in una terminologia "neutrale» che dominò in più capitoli per permettere la condanna e la dettagliata analisi degli effetti negativi del maccartismo nel secondo capitolo.

Il confronto tra Personal influence e The academic mind mostra che l'oggetto e l'epoca in cui viene pubblicata una ricerca insieme al tipo di committente incidono sulla sua valutazione indipendentemente dalla validità dei metodi applicati e delle interpretazioni.

\subsection{I modelli matematici adattivi}

Lo scenario dei modelli matematici adattivi che si dividono nelle due grandi famiglie dei Sistemi evolutivi e delle Reti neurali artificiali (Artificial Neural Network - ANN) è molto distante da quello delle relazioni tra matematica e sociologia proposte da PFL. Per entrare in questo nuovo scenario può essere utile raccontare l'inizio di una di queste famiglie: quella delle ANN.

La storia delle ANN è composta da quattro fasi: (i) preliminari; (ii) fase di espansione all'interno degli studi sull'intelligenza artificiale; (iii) fase di delusione; (iv) fase di espansione con applicazioni delle ANN in tutte le direzioni. 
La ricostruzione dei preliminari può essere in parte soggettiva. Dato che si parla di intelligenza artificiale mi piace inserire tra i contributi preliminari quello di Edgar Allan Poe (1836) che analizzò un falso automa (il giocatore di scacchi di Kempelen) indicando le differenze tra l'intelligenza umana e quella artificiale. Contributi preliminari meno soggettivi sono invece quelli di Alan Turing e di Norbert Wiener.

Steve J. Heims ${ }^{31}$ ha precisato che che quando Wiener organizzò nel 1946 a New York le prime conferenze sulla cibernetica e scienze sociali con i finanziamenti della Macy Foudation invitò anche PFL che partecipò però a un solo incontro mostrandosi poco interessato a questo filone di analisi che doveva portare alle ANN mentre questi seminari incontrarono la massima attenzione da parte di Gregory Bateson. C'è quindi stata una frattura esplicita tra la matematica per le scienze sociali di PFL e la matematica che porta alle ANN. Tra i preliminari più specifici delle ANN si possono ricordare quelli del neurofisiologo, Warren Mac Culloch e del matematico Walter Pitts che nel 1943 scrissero un paper su come i neuroni potrebbero funzionare presentando un modello di ANN con circuiti elettrici. Nel 1948 W. Ross Ashby pubblicò l'articolo "Design for a brain» e nel 1949 Donald Hebbe pubblicò il suo libro The organisation of behavior in cui venne presentata una legge sull'apprendimento dei neuroni (The Hebbian learning law).

La fase di espansione delle ANN può essere fatta iniziare nel 1951. Nel 1951 uscì il romanzo Foundation di Isaac Asimon in cui lo psicostorico Hari Seldon presentava una nuova era: l'evoluzione della società è umana che, ha elementi di imprevedibilità ma nel suo insieme segue leggi matematiche purché a un adeguato livello di complessità. Nello stesso anno, Marvin Minski, che lavorava a Princeton, creò la prima ANN. Nell'estate del 1956 si svolse il congresso di Dartmouth sulla Intelligenza artificiale (al congresso fatto presso il Darmouth College di Hanover parteciparono matematici, ingegneri e psicologi). Nel 1957 venne creato da Frank Rosenblatt alla Cornell University il Perceptron. Nel 1959 Bernard Widrow e Marcian Hoff a Stanford svilupparono due modelli chiamati Adeline e MADALINE (dal nome Multiple ADAptive LINear Elements). Nel 1960 Rosenblatt pubblicò il suo libro Principles of neurodynamics about modelling the brain e nello stesso anno venne realizzato un Multi-Layer Perceptron (MPL).

A questa fase di entusiasmo per le ANN seguì una fase di disillusione. Nel 1968 usci il fim 2001: A space Odyssey di Stanley Kubrick che era una esaltazione della Intelligenza artificiale ma anche una individuazione dei rischi relativi. Nel 1969, in cui arrivò il primo uomo sulla luna, Marvin Minsky e Seymour Papert pubblicano il libro Perceptrons in cui misero in evidenza

31 Heims, 1991. 
i limiti della ANN costruita da Rosenblatt. Il libro ebbe una grandissima influenza e sembrò segnare la fine delle ANN. Negli anni '70 le ANN continuarono a essere studiate da ricercatori isolati (nel 1971 Crossberg inizia a pubblicare i suoi studi sulle ANN non lineari, nel 1972 Kahonen individuò il Learning vector quantization, nel 1974 Werbos presenta il Back-propagation algoritm ecc.).

La seconda fase di espansione avvenne negli anni ' 80 e tra i protagonisti di questa ripresa di interesse per le ANN emerse il fisico John Hopfield che nel 1982 presentò alla National Academy of Science il saggio Neural networks and physical systems with emergent collective computational abilities; nel 1982 Kahonen presentò la Self Organizing Map (SOM); nel 1983 Carpenter e Grossberg la Adaptive Resonance Theory (ART); nel 1984 venne presentata la Boltzmann Machine; nel 1986 Rumelhart e McClelland editarono il libro Parallel distributed processing. Explorations in the microstructure of cognition; nel 1988 Brumhead e Lowe presentarono la Radial Basis Function (RBF) ecc.

Alla fine di questa fase il cambiamento fu radicale: le ANN si affermano come una classe di potenti algoritmi matematici che uscirono dal dibattito sulla Intelligenza artificiale (matematica, ingegneria, psicologia, biologia) per diventare algoritmi autonomi utilizzabili in tutte le scienze sociali. I cambiamenti tecnologici nei computers permisero di utilizzare programmi di ANN su i personal computers e questi programmi permisero di fare previsioni all'interno di discipline diverse come la sociologia, l'economia, la medicina ecc.

Parallelamente a questa storia ci sono altre storie come quella della fuzzy logic (la logica sfumata) ${ }^{32}$ che rende più complessi i livelli con cui le variabili possono essere studiate e poi la storia delle nuove acquisizioni matematiche che permettono di considerare contemporaneamente variabili a livelli diversi di scale, la possibilità di passare da modelli lineari a modelli non lineari con la possibilità di far selezionare al computer i modelli non lineari più adeguati, la possibilità di considerare relazioni di feedback tra variabili ecc.

Queste aumentate possibilità portano allo sviluppo dei modelli matematici adattivi proposti e sperimentati da Massimo Buscema, amico e condirettore di Quality and Quantity da più di venti anni, che li ha elaborati con il suo Gruppo del Semeion, il Centro studi di Roma sul cui sito (www. semeion.it) è possibile avere tutte le informazioni e punti di riferimento bibliografici.

Buscema definisce questa classe generale di modelli matematici «modelli matematici adattivi» perché i processi naturali sono complessi e adattivi e i sistemi artificiali che cercano di emularli sono costruiti in modo da essere

32 Per una descrizione della storia della fuzzy logic rinvio a Capecchi, 1996, pp. 61-66. 
adattivi. Questa classe generale di modelli si articola in due grandi famiglie: quella dei sistemi evolutivi e le reti neurali. I sistemi evolutivi sono sistemi che si evolvono a partire da regole e da vincoli che vengono loro imposti e devono trovare una organizzazione ottima rispetto a un determinato obiettivo. Le reti neurali invece sono invece modelli matematici che attraversano una fase di «apprendimento» costruendosi autonomamente una serie di «stati interni». La rete neurale, come afferma Buscema, si fa una rappresentazione interna dell'ambiente e se viene aiutata con degli algoritmi evolutivi adeguati può ottimizzare il suo apprendimento e raggiungere la previsione desiderata. Tra gli stati di input e quelli di output ci sono così degli stati «nascosti» (hidden) degli «stati interni» della rete. Vi sono così dei nodi di input, dei nodi hidden e dei nodi output:

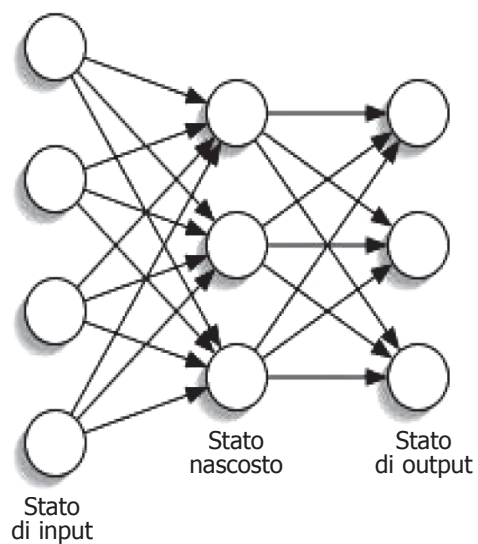

Per chiarire quanto affermato facciamo alcuni esempi di ricerca realizzati da Massimo Buscema e dal suo Semeion Group. Il punto di partenza è come riuscire a effettuare una previsione e si possono considerare due applicazioni: una per riuscire a prevedere se una persona avrà o meno probabilità elevate di contrarre il morbo di Alzheimer ed una per riuscire a prevedere chi, in una classe di studenti, ha forti probabilità di essere bocciato e andare incontro a un insuccesso scolastico. In entrambe le applicazioni si tratta di realizzare una previsione e già questo tipo di precisare il punto di partenza è diverso dallo scenario dei modelli causali lineari di PFL dove ciò che si proponeva era una metodologia per arrivare a una spiegazione. Tra spiegazione e previsione ci sono certamente dei collegamenti ma, come dovrebbe risultare abbastanza evidente dalle due applicazioni che mi propongo di illustrare, si tratta di due concetti diversi. 


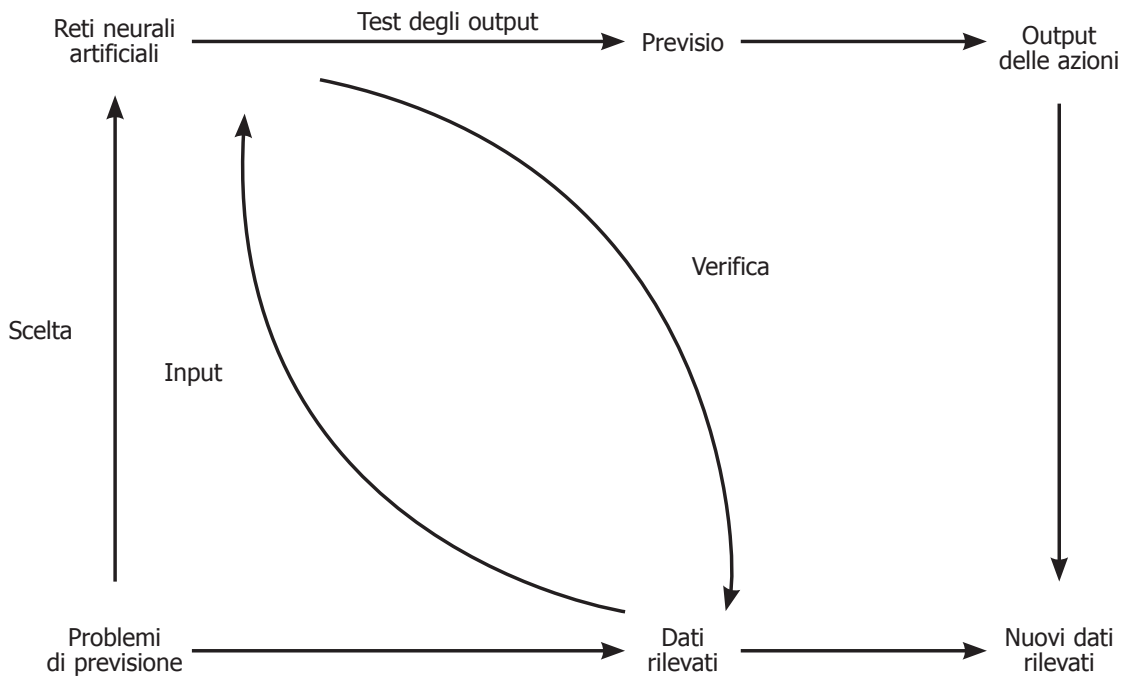

Come risulta dalla figura sopra indicata ${ }^{33}$ il punto di partenza è un problema di previsione (in questo caso la previsione che una data persona possa essere colpita dall'Alzheimer o da una bocciatura) e le prime fasi che devono essere realizzate sono due; (a) la scelta del tipo di ANN idonea per questo tipo di problema; (b) la raccolta dei dati da fornire come input alla rete artificiale.

Per quanto riguarda scelta del tipo di ANN per l'applicazione in campo educativo coordinata da Vincenzo Carbone e Giuseppino Piras ${ }^{34}$ è stata scelta una Rete Feed Forward Supervisionata mentre per quanto riguarda le applicazioni relative all'Alzheimer sono state definite strategie diverse a seconda delle sperimentazioni effettuate ${ }^{35}$.

In quanto ai dati raccogliere, nell'applicazione in campo educativo è stato realizzato un questionario di 224 items che permetteva di individuare le differenze tra il gruppo degli studenti best e quelli drop out mentre nella applicazione per prevedere l'Alzheimer sono stati utilizzate le tracce degli elettroencefalogrammi a persone che avevano o non avevano l'Alzheimer.

Dopo queste due prime fasi (scelta delle ANN e scelta dei dati) questi dati sono fatti apprendere dalla ANN (terza fase) per cui si ha una relazione

33 La figura è presente in Capecchi, 2010, p. 67.

34 Carbone \& Piras, 1999.

35 Queste strategie sono tutte esposte nel sito del Semeion www.semeion.it nella sezione Ricerca applicata / biomedicina. 
circolare tra ANN e dati e, dopo avere testato gli output possibili (quarta fase), si può effettuare la previsione (quinta fase) i cui risultati possono portare a una Nuova raccolta di dati (sesta fase).

È importante comprendere bene questa sequenza. Nella applicazione in campo educativo il questionario viene somministrato all'inizio dell'anno e a giugno è possibile sapere, a seconda dei risultati che gli studenti di quella classe hanno ottenuto, quale sia stato il gruppo degli studenti best e quale quello degli studenti drop out. Questi dati, compresa la separazione tra i due gruppi, sono fatti apprendere dalla ANN e viene fatto un primo test di questi risultati per verificare la possibilità della ANN di discriminare tra i due gruppi. La previsione viene effettuata all'inizio dell'anno scolastico successivo. Gli studenti compilano lo stesso questionario e sulla base dei risultati dell'anno precedente la ANN indica nominativamente quali degli studenti è «destinato» all'insuccesso scolastico oppure no. Questa sequenza mostra la complessità e anche i problemi che possono porre questi modelli di previsione. Supponiamo infatti che questa previsione fatta all'inizio dell'anno sia attendibile. A chi insegna viene offerta la possibilità di conoscere subito chi sono gli studenti best e chi sono i drop out e in quali direzioni sono più deboli (ad esempio in matematica o in altre materie). Diventa allora molto importante che gli insegnanti facciano un uso "corretto" dei dati: si impegnino cioè a fare in modo che la previsione non venga raggiunta e aiutino maggiormente il gruppo dei drop out in modo da non farlo arrivare a una situazione di insuccesso scolastico.

Consideriamo la previsione relativa al morbo di Alzheimer. Anche in questo caso l'équipe medica deve essere in grado di fare un uso "corretto" delle informazioni che la ANN le ha fornito. Di fronte a una persona a cui si dice che ha elevate probabilità di essere colpita dal morbo di Alzheimer è evidente che questa informazione fa accompagnata da una adeguata strategia di «prevenzione» tesa a rallentare l'insorgere della malattia. Sarebbe infatti del tutto assurdo che venisse data l'informazione senza fare una azione adeguata. Si può quindi affermare che queste capacità di previsione delle ANN pongono problemi etici inediti nel mondo dei modelli causali lineari. A chi insegna o a chi è medico viene offerto un potere che può essere usato a fin di bene o in modo negativo come mostra drammaticamente il racconto del $1956 \mathrm{di}$ Philp K. Dick, Minority report (Rapporto di minoranza) da cui è stato tratto il film di Steven Spielberg del 2002.

Per comprendere meglio le distanze di questi modelli matematici adattivi rispetto a quelli di PFL viene affrontata la relazione, che è centrale per capire questo tipo di modelli, tra previsione e spiegazione.

Questi modelli matematici adattivi con tutta la loro gamma di differenze interne sono modelli di previsione. In quale modo possono essere valu- 
tati come modelli di spiegazione? La risposta non è scontata. Consideriamo l'applicazione relativa alla previsione dell'Alzheimer. Ė del tutto evidente che l'équipe medica che ha collaborato con Buscema ha scelto come dati di partenza quelli basati su un elettroencefalogramma e questa scelta è stata basata su ipotesi di tipo clinico/medico, sono state cioè definite relazioni «teoriche» (di spiegazione) tra le tracce di un elettroencefalogramma e la presenza di un possibile Alzheimer. Ci si può allora chiedere: il fatto che questi dati utilizzati da una ANN "prevedano» con probabilità elevatissime la possibilità di essere colpiti dal morbo di Alzheimer in quale modo permette di approfondire la ricerca delle cause dell'Alzheimer? La risposta può essere data solo dalla équipe medica che studia l'Alzheimer e che si propone anche di «spiegare» cosa agevola il sopraggiungere di questa malattia. Da un osservatore esterno come chi scrive si può solo osservare che, in questo caso, un potente modello di previsione non è detto sia anche un modello ugualmente potente di «spiegazione».

Una analisi più dettagliata (e più dominabile da chi scrive) può essere fatta in relazione alla applicazione sull'insuccesso scolastico. La ANN utilizza in questo caso l'intero pacchetto delle risposte alle 224 items come un potente strumento di previsione da affidare agli insegnanti del corso successivo. Questa esperienza non è stata portata avanti ma supponiamo che lo fosse quali relazioni avrebbero potuto essere allora definite tra "previsione»e "spiegazione»?

In questo caso la sperimentazione è diversa da quella dell'esempio precedente per tre principali motivi: (a) il «pacchetto» di items è variabile e dipende dal ricercatore; (b) è possibile agire didatticamente su i ragazzi e le ragazze in difficoltà individuate dalle ANN; (c) i risultati della ANN permettono non solo di identificare le ragazze e i ragazzi a rischio ma anche di verificare la maggiore vicinanza lontananza delle 224 items dalla variabile dipendente «insuccesso scolastico».

Si può allora verificare quale «spiegazione» teorica venga suggerita dalla maggiore vicinanza/lontananza delle items alla variabile «insuccesso scolastico" e si può cercare di cambiare il pacchetto delle items per verificare la possibilità di una migliore spiegazione. Inoltre gli insegnanti, che all'inizio del corso successivo trovano identificati dalla ANN i soggetti più «a rischio», possono condurre una analisi più qualitativa su questi soggetti e verificare quali sono le strategie possono essere portate avanti su di loro per portare questi ragazzi ad amare maggiormente la matematica o le materie che, dalle loro riposte al questionario, risultano essere meno facilmente apprendibili e quindi più agevolanti la loro bocciatura. Ed è del tutto evidente che il successo/insuccesso delle loro azioni porta ad arricchire una «spiegazione» teorica dell'insuccesso scolastico. 
Ciò che emerge è che siamo nel pieno di un ricerca che adotta il paradigma della ricerca azione. L'obiettivo del ricercatore e degli insegnanti di una data classe è quello di fare in modo che nessun ragazzo o ragazza vada incontro a una bocciatura e questo risultato può essere raggiunto attraverso due vie: (a) la conoscenza della previsione della ANN che permette di concentrare subito, all'inizio dell'anno, l'attenzione su le ragazze e i ragazzi «a rischio» essendo poi campito di chi insegna capire quali azioni possano essere realizzate per renderli più forti nelle diverse materie; (b) conoscenza delle relazioni tra le items del questionario e la variabile dipendente «insuccesso scolastico", conoscenza che permette di elaborare, verificare e proporre "spiegazioni» psicologiche e sociologiche dell'insuccesso scolastico.

In questa applicazione delle ANN la relazione "previsione»/«spiegazione» va vista all'interno di una sequenza di ricerche/azioni che prosegua per più anni scolastici. C'è una ricerca di «spiegazione» che dipende dalle ricerche: $\mathrm{i}$ risultati del primo questionario (e questionari successivi eventualmente modificati) analizzati in termini di vicinanza /lontananza delle items dalla variabile «dipendente» insuccesso scolastico. E c'è una ricerca di «spiegazione» basata sulle azioni degli insegnanti per fare in modo che le ragazze e i ragazzi drop out possano superare le loro difficoltà ed evitare di essere bocciati. La "previsione", in questo tipo di applicazione, si intreccia molto bene con la «spiegazione» perché, anche se i due termini sono giustamente tenuti distinti, fanno insieme parte di uno stesso progetto di ricerca/azione.

I nuovi orizzonti dei metodi quantitativi invitano quindi a riprendere riflessioni metodologiche più ampie sulla relazione previsione/spiegazione e a rendere maggiormente consapevoli delle scelte fatte nei paradigmi della ricerca.

\section{RIFERIMENTI BIBLIOGRAFICI}

Alberoni, F. (a cura di). (1967). L'attivista di partito. Bologna: Il Mulino.

Berelson, B., Lazarsfeld, P. F., \& Mc Phee, W. N. (1954). Voting: A study of opinion formation in a presidential campaign. Chicago, IL: University of Chicago Press.

Blalock, H. (Ed.). (1985). Causal models in the social sciences. New York: Aldine de Gruyter.

Capecchi, V. (1967). Metodologia e ricerca nell'opera di P.F. Lazarsfeld. In Introduzione all'opera di P.F. Lazarsfeld. Metodologia e ricerca sociologica (pp. 7-184). Bologna: Il Mulino.

Capecchi, V. (1985). Appunti per una riflessione sulla metodologia della ricerca sociologica. Quaderni di Sociologia, 4-5, 112-169. 
Capecchi, V. (1996). Tre Castelli, una Casa e la Città inquieta. In C. Cipolla \& A. De Lillo (a cura di), Il sociologo e le sirene (pp. 37-99). Milano: Franco Angeli.

Capecchi, V. (1999). Il contributo di Lazarsfeld alla metodologia sociologica. Sociologia e Ricerca Sociale, 58-59, 35-82.

Capecchi, V. (2010). Mathematics and sociology. From Lazarsfeld to artificial neural networks. In V. Capecchi, M. Buscema, P. Contucci, \& B. D’Amore (Eds.), Applications of mathematics in models, artificial neural networks and arts (pp. 1-78). Dordrecht: Springer (trad. it., Matematica e sociologia. Da Lazarsfeld alle reti neurali artificiali. Sociologia e Ricerca Sociale, 2008, 87, 5-90).

Capecchi, V. (2013). I tre paradigmi della ricerca sociologica. Quaderni di Sociologia, $62,39-54$

Carbone, V., \& Piras, G. (1999). Orientamento e dispersione scolastica: le reti neurali artificiali come supporto dell'azione formativa. In M. Buscema \& Semeion Group (a cura di), Reti neurali artificiali e sistemi sociali complessi. Teoria-modelli applicazioni, Vol. 1: Teoria e modelli (pp. 170-200). Milano: Franco Angeli.

Conte, R., Hagelmann, R., \& Terna, P. (Eds.). (1997). Simulating social phenomena. Berlin: Springer.

Galli, G. (a cura di). (1968). Il comportamento elettorale in Italia. Bologna: Il Mulino. Goffman, E. (1961). Asylums (trad. it., Torino: Einaudi, 2003).

Granovetter, M. S. (1973). The strength of weak ties. American Journal of Sociology, 6, 1360-1380.

Harding, S. (1992). After the neutrality ideal: Science, politics and strong objectivity. Social Research, 59, 567-588.

Harding, S. (2004). Rethinking standpoint epistemology. What is strong objectivity? In S. Nagy Hesse-Biber \& M. L. Yaiser (Eds.), Feminist perspectives on social research (pp. 49-82). New York: Oxford University Press.

Heims, S. J. (1991). The cybernetics group. Cambridge, MA: MIT Press (trad. it., I cibernetici. Un gruppo e un'idea. Roma: Editori Riuniti, 1994).

Katz, E., \& Lazarsfeld, P. F. (1956). Personal influence: The part played by people in the flow of mass communications. New York: Free Press (trad. it. a cura di M. Morcellini, L'influenza personale in comunicazione. Roma: Armando, 2012).

Lazarsfeld, P. F. (1986). Prefazione. In M. Jahoda, P. F. Lazarsfeld, \& H. Zeisel, Marienthal: The sociography of an unemployed community. London: Tavistock, 1971 (trad. it., I disoccupati di Marienthal. Roma: Edizioni Lavoro).

Lazarsfeld, P. F., Berelson, B., \& Gaudet, H. (1944). The people's choice: How the voter makes up his mind in a presidential campaign. New York: Columbia University Press.

Lazarsfeld, P. F., \& Neal, H. H. (1968). Latent structure analysis. Boston: Hougton Miffin. 
Lewin, K. (1946). Action research and minority problema. Journal of Social Issues, 2, 34-46; ripreso in K. Lewin (Ed.), Resolving social conflict: Selected papers on group dynamics (pp. 205-206). New York: Harper.

McClung Lee, A. (1970). On the context of relevance. In G. Jacobs (Ed.), The partecipant observer. Encounters with social reality. New York: Geoge Braziller.

Neil, W. H. (1999). Latent structure analysis at fifty. Paper presented at the 1999 Joint Statistical Meetings, Baltimore.

Ramazanoglu, C., \& Holland, J. (2002). Feminist methodology: Challenges and choices (pp. 138-139). Thousand Oaks, CA: Sage.

Reinharz, S. (1992). Feminist methods in social research. New York: Oxford University Press.

Vezzoni, C., \& Mancosu, M. (2013). The strength of weak political ties. The case of Movimento 5 Stelle. Relazione presentata alla Seconda Conferenza Europea sulla Ricerca Elettorale Comparata, Rodi.

\section{RiassunTO}

In questo saggio le nuove prospettive dei metodi quantitativi sono analizzate confrontando i modelli causali lineari diffusi nella metodologia proposta da P.F. Lazarsfeld e i modelli adattivi (con i modelli delle "Artificial Neural Networks") oggi diffusi in Italia da Massimo Buscema e dal suo Semeion Group di Roma. Questa analisi dei metodi quantitativi è esaminata tenendo presente tre paradigmi (Kuhn) delle ricerca sociologica: (a) il paradigma della oggettività; (b) il paradigma della ricerca azione; (c) il paradigma della "Feminist methodology". In questo saggio è presentata una mappa delle relazioni fra i tre paradigmi in termini di specificitàlgeneralità e una ricerca in area educativa in cui viene applicata una ANN che permette una riflessione sulle relazioni tra spiegazione e previsione. Le differenze tra i modelli causali lineari e le reti neurali artificiali sono spiegate come: differenze in modelli lineari / non lineari, differenti possibilità di utilizzare variabili a diversi livelli di scala, differenti paradigmi nella ricerca sociale, differenze nella relazione tra previsione e spiegazione, differente uso del tempo nel processo della ricerca.

Parole chiave: Metodologia femminista, Modelli causali lineari, Paradigma dell’oggettività, Reti neurali artificiali, Ricerca-azione. 